\title{
Experimental investigation of strength properties of 3D printed ABS composites
}

\author{
Abdul Quader Shurjeel ${ }^{1 *}$, Narendra Pothula $^{2}$, Eshwaraiah Punna ${ }^{3}$ \\ ${ }^{1}$ VNR Vignana Jyothi Institute of Engineering \& Technology, Mechanical Engineering Department, Hyderabad,Telangana, India \\ ${ }^{2}$ VNR Vignana Jyothi Institute of Engineering \& Technology, Mechanical Engineering Department, Hyderabad,Telangana, India \\ ${ }^{3}$ GITAM Deemed to be University, Mechanical Engineering Department, Hyderabad,Telangana, India 502329
}

\begin{abstract}
Owing to the huge demand and dependency of the industry on the lightweight and superior mechanical properties products as well as components, the materials like CF-ABS (Acrylonitrile Butadiene Styrene reinforced with carbon fibers) and PC-ABS (Acrylonitrile Butadiene Styrene reinforced with polycarbonate) have gained utmost importance in the current scenario. The present research in this paper focuses on finding the mechanical properties, mainly the tensile, compression, and flexural properties of both the above-said materials. FDM (Fused Deposition Modelling) is used as the printing technique in this research as it is the most suitable and widely used for the selected materials. After experimentation, a comparison was made between the two materials, and it is found that the PC-ABS material is stronger in compression, tension as well as in flexural at all the parametric settings. The infill percentage was observed to be proportional to the strength of the material as expected. Triangular infill geometry was more strong in compression and flexural whereas grid infill geometry was strong in tension. produced stronger mechanical properties were observed for 0-degree raster angle in all the three criteria compared to the 45 and 90 -degree raster angles. When the variation of the strength of the material with the infill geometry was observed, the infill geometry was more sensitive in compression and flexural compared to that in tension. The load vs. displacement curves have been plotted to depict the maximum load and the behavior of the material in the elastic and plastic regions.
\end{abstract}

\section{Introduction}

Fused deposition modeling (FDM) is a well-known technique under the Additive manufacturing technology developed by Stratasys company in around 1992[1]. FDM technique produces layer upon layer parts with desired and complex geometries directly from a CAD model usually by the deposition of melt material through the extruder which is the nozzle as opposed to subtractive manufacturing methodologies. There are numerous benefits of the FDM technique, such as design versatility to produce complex geometries without the need for moulds and dies. Internal features of the parts are produced through computer ability, which is impossible in the traditional manufacturing process[2]. When compared with the conventionally manufactured products, Pure plastic products exhibit lower mechanical properties. To address this issue modification in the printing parameters is one approach and another one is the development of material with superior properties. The development of materials with superior mechanical properties is done by blending polymers[3].

In the industries, carbon fiber(CF) is a chief competitor. When carbon fiber is added to ABS with ABS as the base material in FDM, an enhancement in the mechanical properties was observed as compared to the pure plastic parts. The motivation for choosing the CFABS composite is due to its vast applications in many fields such as (medical, sports, automobile, and aviation)[4]. On the other side, PC-ABS composite gives better mechanical behavior in dynamic loading conditions it combines impact resistance and heat of PC and fine processability of ABS. The motivation for choosing PC-ABS composite is due to its vast applications in many fields such as( dashboards, automobile steering wheel covers) NASA used PC-

\footnotetext{
* Corresponding author: shurjee1998@gmail.com
} 
ABS printed parts in rover which was lightweight and durable[5].

The effect of FDM parameters was examined by $\mathrm{M}$. Kamaal et al[2021 on the mechanical properties of the material. They have observed the influence parameters such as layer thickness, building direction, and infill density on the tensile strength and concluded that the superior strength was observed when layer thickness was $0.25 \mathrm{~mm}$ with the infill density of $80 \%[6]$. Sridharan Kannan et al [2020] have examined the strength of 3 different materials (ABS, PC, PC-ABS) with FDM parameters as infill percentage- $100 \%$, layer height of $0.1 \mathrm{~mm}$, and raster angle of $(45,-45)$ and concluded that PC-ABS composite exhibits superior elastic modulus and load-carrying ability than ABS \&PC materials[7]. Effect of FDM parameters like orientation, layer height, the temperature of the extruder tip, speed of printing on the mechanical properties of fabricated CF-based plastics parts was studied by Fuda Ning, Weilong, yingbin et al[2017]. They concluded that orientation of $[0,90]$ showed considerably superior mechanical properties than raster angle of $[-45,45]$ with $25 \mathrm{~mm} / \mathrm{s}$ infill speed, $200^{\circ} \mathrm{C}$ nozzle temp, $0.25 \mathrm{~mm}$ layer thickness[8]. Cantrell et al[2017] considered the different printing directions for polycarbonate plastic and concluded that on edge printing orientation will give maximum tensile strength[9]. Layer height, printing direction, and infill orientation were considered by Uddin et al[2017] to evaluate the mechanical properties for $3 \mathrm{~d}$ printed ABS material. They have concluded that raster orientation has a very minute effect on compressive properties than the tensile properties[10]. Effect of infill geometry and the infill percentage were considered by Miguel Fernandez-Vicente et al [2016] to cut down the material utilization, printing time and performed the experimental study by varying three infill patterns with three infill densities (20\% 50\% 100\%). The researchers have observed that $100 \%$ infill density produces superior tensile strength[11]. Rankouhi et al[2016] have observed the Layer height and orientation effect on mechanical properties combined with statistical analysis and showed that lower layer height produces stronger components[12]. K.G. Jaya Christiyan et al[2016] examined the mechanical properties of ABS + hydrous magnesium silicate composite material by considering different parameters as layer height, nozzle diameter, and speed. They have concluded that maximum tensile and flexural strength at a layer height of $0.2 \mathrm{~mm}$ and speed of $30 \mathrm{~mm} / \mathrm{s}[13]$. The mechanical behavior was observed to be more sensitive towards printing orientation when compared to the raster angle [14]. Hill et al[2014] considered different raster angles on PC $3 \mathrm{~d}$ printed specimens and concluded that the 0 -degree raster angle gives maximum tensile strength than 90 degrees [15].

The research in this paper investigates the mechanical properties of ABS-composites produced by FDM printers with different parameters to produce superior 3Dprinted products for tensile strength, compressive strength, and flexural strength. A summary of the process parameters, mechanical tests performed, different materials used, the effect of process parameters variations on test results are mentioned in the next section.

\section{Experimental details}

\subsection{Materials and methods}

The materials used in this study are CF-ABS and PCABS filaments of $1.75 \mathrm{~mm}$ dia, $5.50 \%$ carbon fiber, and PC produce by WOL 3D, India Fig. 1. AKAR 600Pro 3D printer was used to print the specimens of both materials. Specimens modelling was done in Solidworks, software Cura Ultimaker for 3Dprinting. Tensile, Compression, Flexural test are performed on MCS-Universal Testing Machine Fig.5.

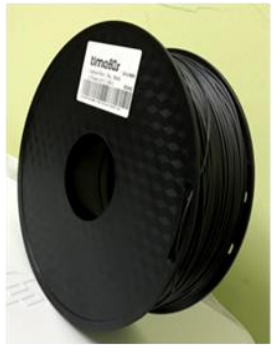

(a)

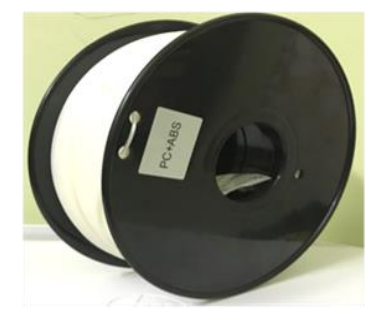

(b)
Fig. 1: Composite spools (a) CF-ABS (b) PC-ABS

\subsection{Sample preparation}

To evaluate the mechanical properties of ABS-based plastic composite material, modelling of the different geometries was performed according to American Society Of Testing Materials(ASTM) standards ASTM D638 for tensile test, ASTM D695 for the compression test, and ASTM D790 for flexural test Fig. 2.

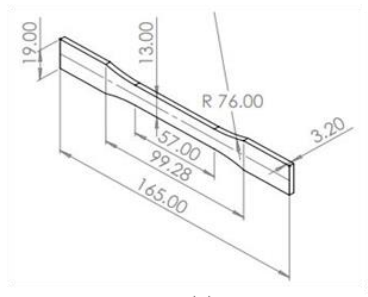

(a)

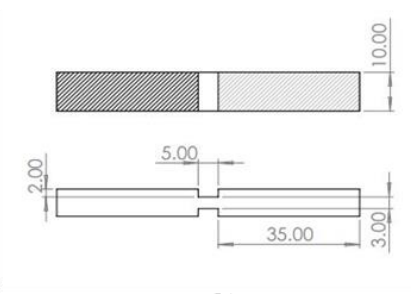

(b)
Fig. 2. Dimensions of (a) ASTM D638 (b) ASTM D695 (c) ASTM D790 


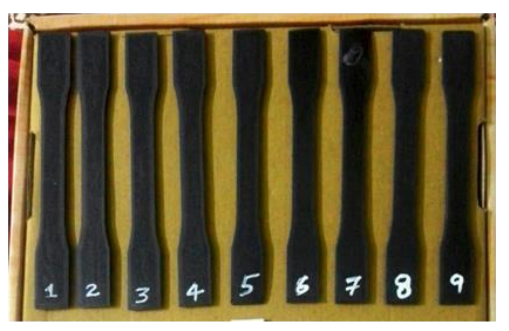

(a)

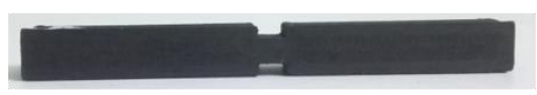

(b)

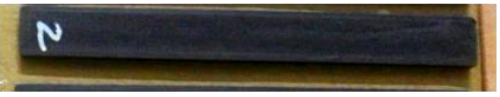

(c)

Fig. 3. FDM Printed specimens of (a) ASTM D638 (b) ASTM D695 (c) ASTM D790

\subsection{Experimental setup}

The present study mainly focuses on three process parameters of FDM that affect the mechanical properties, which are Infill geometries, Raster angle, and Infill percentage. The influence of the above-stated printing parameters on tensile, compressive, and flexural strengths were independently studied.

Infill geometry is the $3 \mathrm{D}$ structure of the material printed inside of a part which affects the part strength, printing time, and weight. The infill geometries selected in this study are Grid, Triangle, Tri-Hexagonal. Raster angle is the deposition of filament according to the raster angle tool path with respect to the $\mathrm{X}$-axis of the build table. The raster angles selected in this study are $0^{\circ}, 45^{\circ}$, $90^{0}$ Fig. 4. Infill density shows the compactness of the components where $100 \%$ means solid and $0 \%$ means shell. In this study, three infill percentages are considered i.e $60 \%, 70 \%, 80 \%$.
Infill Geometries

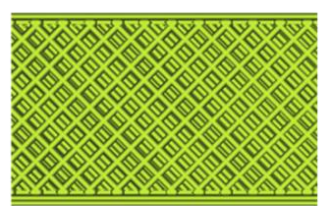

Grid

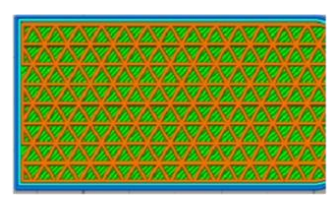

Triangular

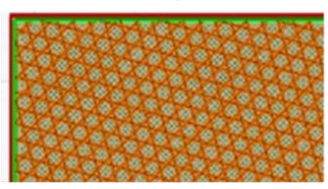

Tri- Hexagonal

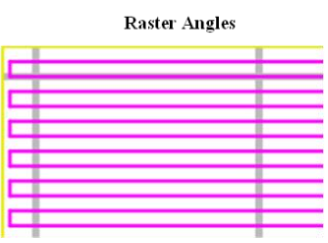

o Degree

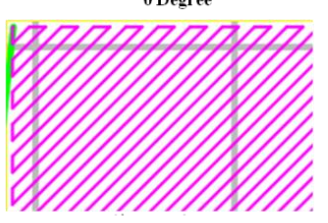

45 Degree

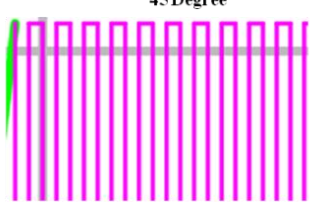

90 Degree
Fig. 4. FDM Printing parameters

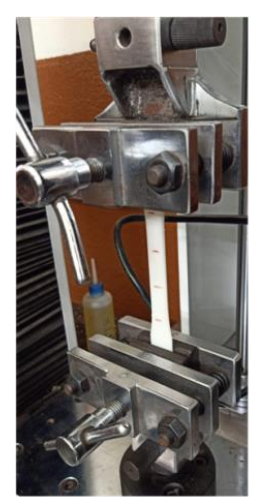

(a)

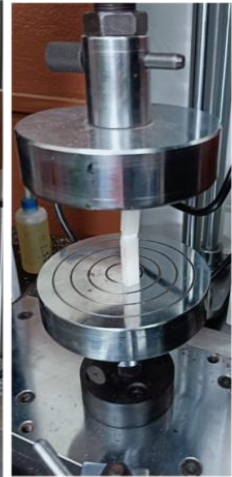

(b)

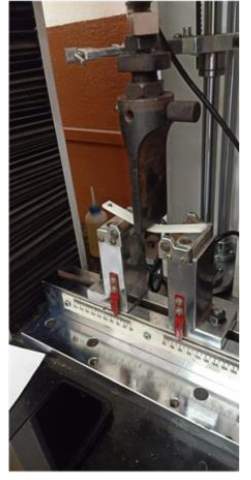

(c)
Fig. 5. Testing of specimens (a) Tensile (b) Compressive

(c) Flexural

Table 1. FDM printing parameters

\begin{tabular}{|c|c|}
\hline Materials & $\begin{array}{c}\text { a)CF-ABS } \\
\text { b)PC-ABS }\end{array}$ \\
\hline Diameter & $1.75 \mathrm{~mm}$ \\
\hline Infill geometries & Grid, Triangular, Tri-hexagonal \\
\hline Raster Angles & $0^{0}, 45^{0}, 90^{\circ}$ \\
\hline Infill Percentage & $60 \%, 70 \%, 80 \%$ \\
\hline Layer height & $0.2 \mathrm{~mm}$ \\
\hline Nozzle & $240^{\circ} \mathrm{C}$ for CF-ABS \\
temperature & $250^{\circ} \mathrm{C}$ for PC-ABS \\
\hline Bed temperature & $80^{\circ} \mathrm{C}$ for CF-ABS \\
& $100^{\circ} \mathrm{C}$ for PC-ABS \\
\hline
\end{tabular}

The details present in Table 1 were used for the experimentation and 9 specimens each for tensile, compression, and flexural for each material were printed at different FDM parameters.

\section{Results and Discussion}

\subsection{Effect of FDM parameters on the tensile strength}

Table 2 shows the different FDM parametric combinations and variation of maximum load and the ultimate tensile strength (UTS) and ultimate compressive strength (UCS) at different parameters. The average of the UTS at three identical parametric settings i.e. at the identical infill geometry, identical infill percentage, and raster angle is shown in the form of a bar diagram in Fig.6. It is evident from the bar graphs that the tensile strength is far more superior for the PC-ABS compared to the CF-ABS composite at all the parametric settings. An approximate two times increase in the tensile strength for PC-ABS compared with the CF-ABS shows that PC-ABS is the go-to composite when the criterion for selection is the 
strength. Furthermore, the variation of tensile strength at varying infill geometry is insignificant for the CF-ABS compared to the PC-ABS. It means the tensile strength is more sensitive to varying infill geometry, infill percentage, and raster angle for $\mathrm{PC}-\mathrm{ABS}$ compared to the CF-ABS composites. Grid infill geometry was observed to be slightly strong compared to the other two infill geometries i.e. triangle and tri-hexagonal.

Table 2. Experimental observations of tensile and compression test

\begin{tabular}{|c|c|c|c|c|c|c|c|c|c|c|c|}
\hline \multirow[b]{2}{*}{ Specimen } & \multicolumn{3}{|c|}{ Process Parameters } & \multicolumn{2}{|c|}{ CF-ABS } & \multicolumn{2}{|c|}{ PC-ABS } & \multicolumn{2}{|c|}{ CF-ABS } & \multicolumn{2}{|c|}{ PC-ABS } \\
\hline & $\begin{array}{c}\text { Infill } \\
\text { geometry }\end{array}$ & $\begin{array}{c}\text { Raster } \\
\text { angle } \\
\text { (degree) }\end{array}$ & $\begin{array}{c}\text { Infill } \\
\text { perce } \\
\text { ntage } \\
(\%)\end{array}$ & $\begin{array}{c}\text { Max Load } \\
\text { (N) }\end{array}$ & $\begin{array}{c}\text { UTS } \\
\text { (MPa) }\end{array}$ & $\begin{array}{c}\text { Max } \\
\text { Load } \\
\text { (N) }\end{array}$ & $\begin{array}{c}\text { UTS } \\
\text { (MPa) }\end{array}$ & $\begin{array}{c}\text { Max } \\
\text { Load } \\
\text { (N) }\end{array}$ & $\begin{array}{c}\text { UCS } \\
\text { (MPa) }\end{array}$ & $\begin{array}{c}\text { Max } \\
\text { Loa } \\
\text { d } \\
\text { (N) }\end{array}$ & $\begin{array}{c}\text { UCS } \\
\text { (MPa) }\end{array}$ \\
\hline 1 & Grid & 0 & 60 & 1178 & 26.45 & 2392 & 55.42 & 589.75 & 18.79 & 2552 & 82.32 \\
\hline 2 & Grid & 45 & 70 & 1268 & 28.82 & 2558 & 57.69 & 603 & 19.15 & 2266 & 74.86 \\
\hline 3 & Grid & 90 & 80 & 1598 & 33.86 & 3374 & 79.74 & 656 & 20.83 & 2868 & 94.84 \\
\hline 4 & Triangle & 0 & 70 & 1276 & 29.46 & 2734 & 61.74 & 755.25 & 24.01 & 3266 & 107.79 \\
\hline 5 & Triangle & 45 & 80 & 1308 & 30.86 & 2638 & 57.01 & 744.25 & 23.69 & 2922 & 90.97 \\
\hline 6 & Triangle & 90 & 60 & 1206 & 28.68 & 2590 & 59.73 & 659 & 21.1 & 2752 & 90.53 \\
\hline 7 & $\begin{array}{c}\text { Tri- } \\
\text { Hexagonal }\end{array}$ & 0 & 80 & 1176 & 27.36 & 2850 & 69.53 & 662.25 & 20.73 & 2774 & 84.78 \\
\hline 8 & $\begin{array}{c}\text { Tri- } \\
\text { Hexagonal }\end{array}$ & 45 & 60 & 1062 & 25.57 & 2242 & 55.24 & 121.2 & 3.76 & 2758 & 85.71 \\
\hline 9 & $\begin{array}{c}\text { Tri- } \\
\text { Hexagonal }\end{array}$ & 90 & 70 & 1142 & 27.24 & 2608 & 63.87 & 115 & 3.81 & 2364 & 76.26 \\
\hline
\end{tabular}

When it comes to the raster angle, the 0 -degree raster angle is far more superior compared to the 45 and 90 degree raster angles. Variation in the Infill percentage has a negligible effect on the individual strength of both the composite materials as it was in the case of the infill geometry. Never the less the higher the infill percentage the stronger is the material in the case of both the composite materials. The load vs. displacement curve for the grid infill geometry with the varying infill percentage for both the composites is shown in Fig.7. It can be observed that the maximum load for the PC-ABS composite is higher when compared with that of the CF-
ABS composite material. When it comes to the variation of maximum load with respect to the infill percentage, the $80 \%$ infill geometry was able to bear the maximum load of 1598 which is significantly higher than the other two infill percentages. There observed an increasing trend of the maximum load with increasing infill percentages as expected. The yield stress was also observed to be behaving the same manner as the ultimate tensile strength for both the material. there observed a $2.18 \%$ elongation for the $60 \%$ infill geometry which is the highest among the three variables i.e. $60 \%, 70 \%$, and $80 \%$ infill densities 


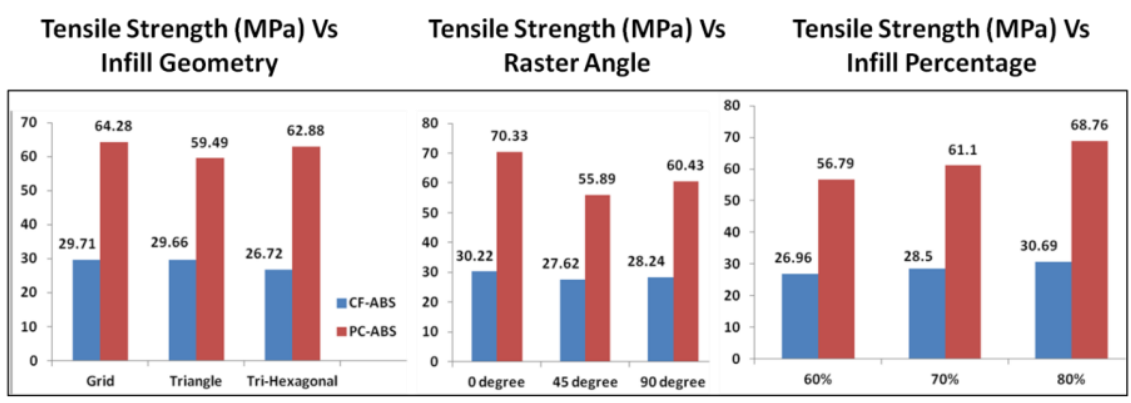

(a)

(b)

(c)

Fig. 6. Bar graph showing the tensile strength Vs. (a) Infill geometry (b) Raster Angle (c) Infill percentage

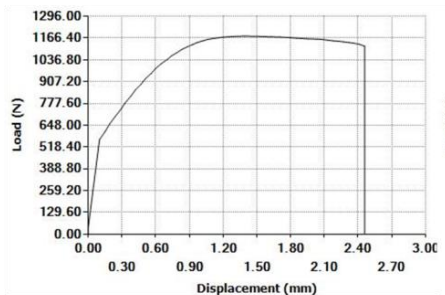

(a)

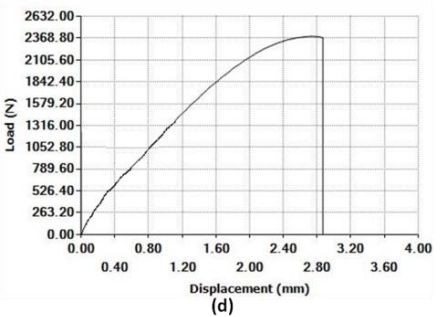

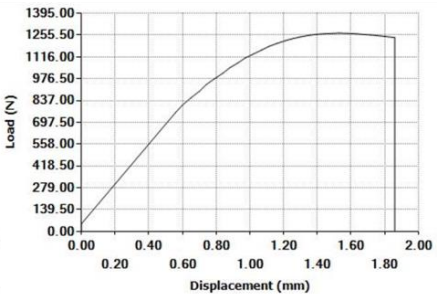

(b)

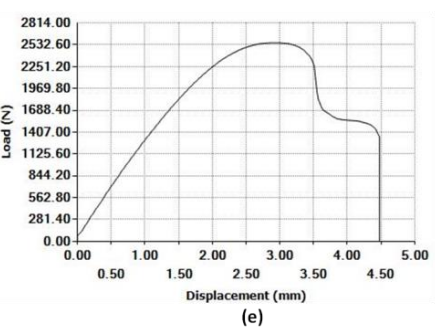

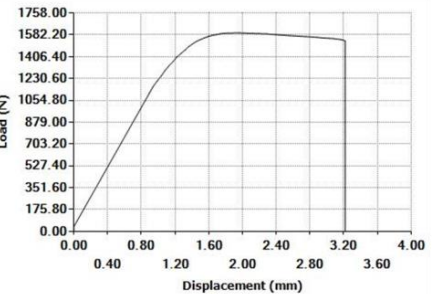

(c)

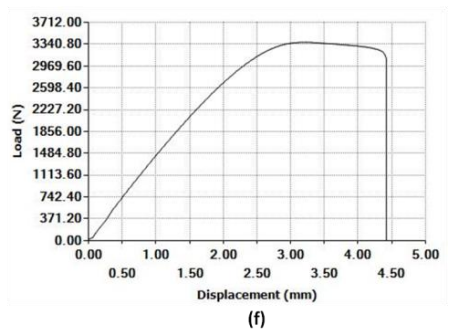

Fig. 7. Load Vs. displacement curves of tensile test for Grid infill geometry with (a-c) CF-ABS (d-f) PC-AB

\subsection{Effect of FDM parameters on the compressive strength}

Table 2 shows the experimental observation of compressive strength and maximum load for CF-ABS and PC-ABS composites. The average compressive strength at a particular infill geometry, raster angle, and infill percentage is shown in Fig. 8. It is evident from Fig.8 that there is a huge difference between the compressive strength of the two composites at almost all the parameters. there observed an approximate five times increase in the compressive strength of the PC-ABS compared to the CF-ABS composite material. As the variation in the compressive strength with infill geometry is significant for both the materials, the compressive strength is more sensitive towards the infill geometry, compared to the other two parameters. 0-degree raster angle showed the maximum compressive strength for both the materials and the compressive strength was proportional to the infill percentage as expected. Fig.9 shows the load vs displacement curve at a constant raster angle with varying infill geometry for both materials. It can be observed that the triangular infill geometry showed the maximum load as well as compressive strength for both the materials compared to the other two infill geometries. The load vs displacement curve for CF-ABS was initially elastic and then become plastic, whereas for $\mathrm{PC}-\mathrm{ABS}$ not elastic region was observed.

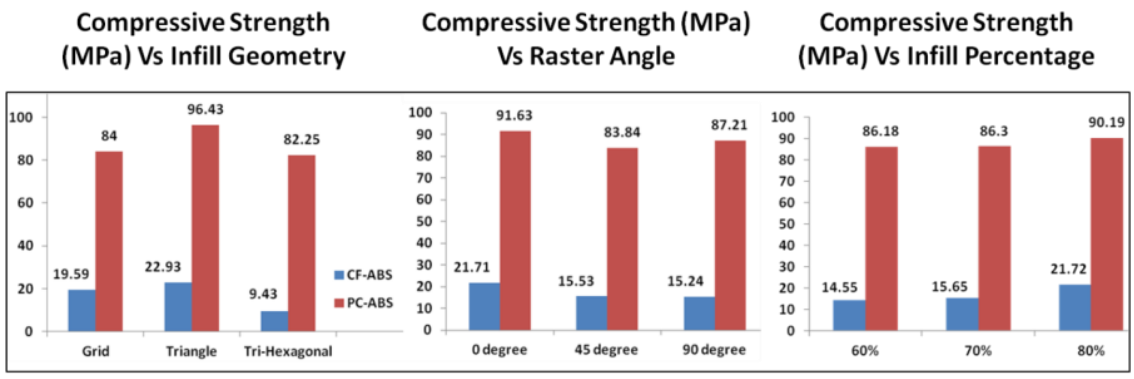

(a)

(b)

(c)

Fig. 8. Bar graph showing the compressive strength Vs. (a) Infill geometry (b) Raster Angle (c) Infill percentage 


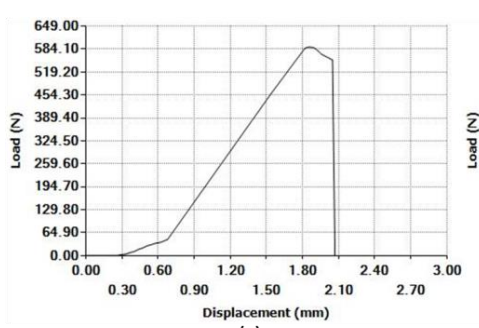

(a)

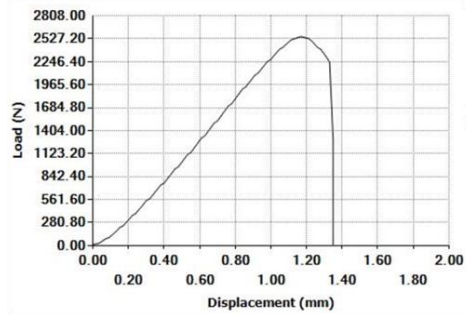

(d)

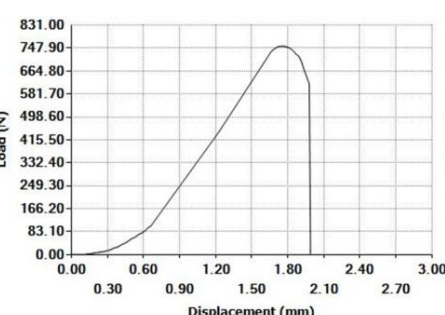

(b) $(\mathrm{mm})$

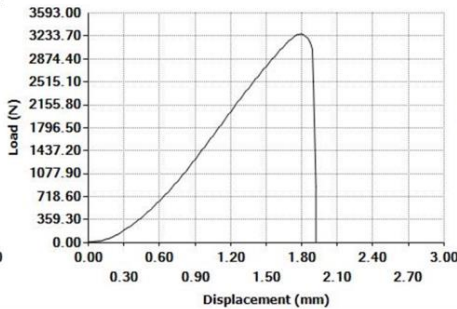

(e)

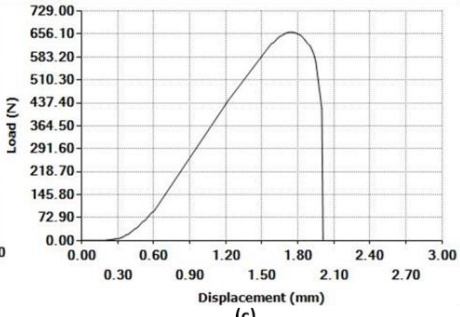

(c)
Displacement (mm)

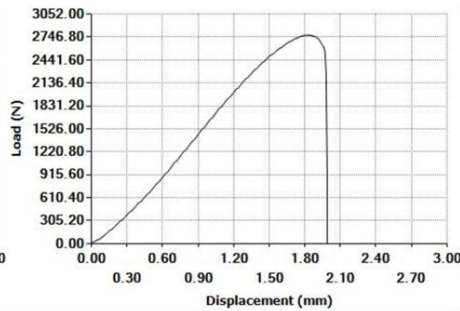

(f)

Fig. 9. Load Vs. displacement curves of compression test for 0-degree raster angle with (a-c) CF-ABS (d-f) PC-ABS

\subsection{Effect of FDM parameters on the flexural}

\section{strength}

Table 3 shows the experimental observations for the flexural or bending test at varying infill geometry, raster angle, and infill percentage. The average variation of the flexural strength at the respective parameters is shown in Fig. 10 in the form of a bar graph. The trend of the CF-ABS as well as the PC-ABS composite material. An approximate two times increase in the flexural strength of the PC-ABS was observed in comparison with the CF-ABS composite material.

Table 3. Experimental observations of flexural test

\begin{tabular}{|c|c|c|c|c|c|c|c|}
\hline \multirow[b]{2}{*}{$\begin{array}{c}\text { Specime } \\
\text { n }\end{array}$} & \multicolumn{3}{|c|}{ Process Parameters } & \multicolumn{2}{|c|}{ CF-ABS } & \multicolumn{2}{|c|}{ PC-ABS } \\
\hline & $\begin{array}{c}\text { Infill } \\
\text { geometry }\end{array}$ & $\begin{array}{c}\text { Raster angle } \\
\text { (degree) }\end{array}$ & $\begin{array}{c}\text { Infill } \\
\text { percentage(\%) }\end{array}$ & $\begin{array}{r}\text { Max } \\
\text { Load } \\
(\mathbf{N})\end{array}$ & $\begin{array}{l}\text { UTS }(\mathbf{N} / \mathbf{m m} \\
\left.{ }^{2}\right)\end{array}$ & $\begin{array}{r}\text { Max } \\
\text { Load } \\
(\mathrm{N})\end{array}$ & $\begin{array}{l}\text { UTS(N/mm } \\
\left.{ }^{2}\right)\end{array}$ \\
\hline 1 & Grid & 0 & 60 & 29.8 & 25.65 & 67.1 & 42.19 \\
\hline 2 & Grid & 45 & 70 & 25.10 & 22.66 & 89.3 & 53.42 \\
\hline 3 & Grid & 90 & 80 & 26.2 & 23.76 & 80 & 47.48 \\
\hline 4 & Triangle & 0 & 70 & 30 & 28.08 & 77.4 & 48.21 \\
\hline 5 & Triangle & 45 & 80 & 27.7 & 25.68 & 80.8 & 47.72 \\
\hline 6 & Triangle & 90 & 60 & 24.5 & 22.33 & 83.5 & 53.36 \\
\hline 7 & Tri-Hexagonal & 0 & 80 & 25.9 & 25.01 & 74.5 & 49.3 \\
\hline 8 & Tri-Hexagonal & 45 & 60 & 5.2 & 4.79 & 12.8 & 9.75 \\
\hline 9 & Tri-Hexagonal & 90 & 70 & 5 & 4.59 & 16.2 & 11.33 \\
\hline
\end{tabular}


Similar to the compressive strength, the flexural strength is superior with triangular infill geometry and 0-degree raster angle. As expected, the flexural strength was observed to be proportional to the infill percentage as was the case for both the tensile as well as compressive strength. The load vs displacement curve at a constant infill percentage of $80 \%$ with varying infill geometry and raster angle is shown in Fig.11. The slope of the elastic region of the load vs displacement curve was higher for the PC-ABS compared to the CF-ABS composite material. Also, the PC-ABS behaved more plastically compared to the CF-ABS material.

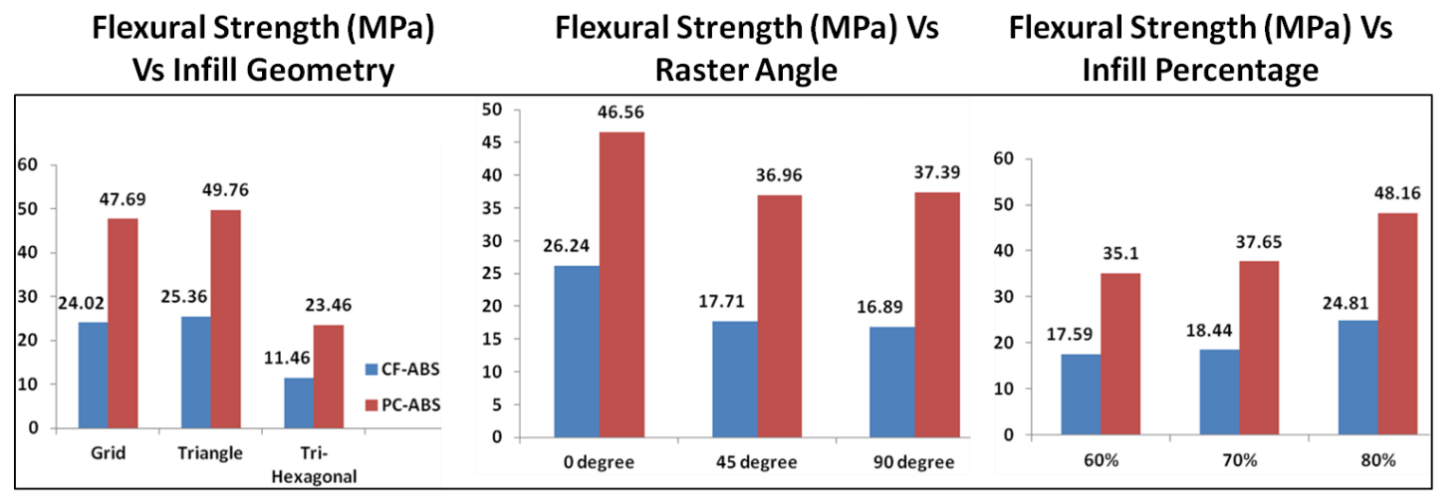

(a)

(b)

(c)

Fig. 10. Bar graph showing the flexural strength Vs. (a) Infill geometry (b) Raster Angle (c) Infill percentage
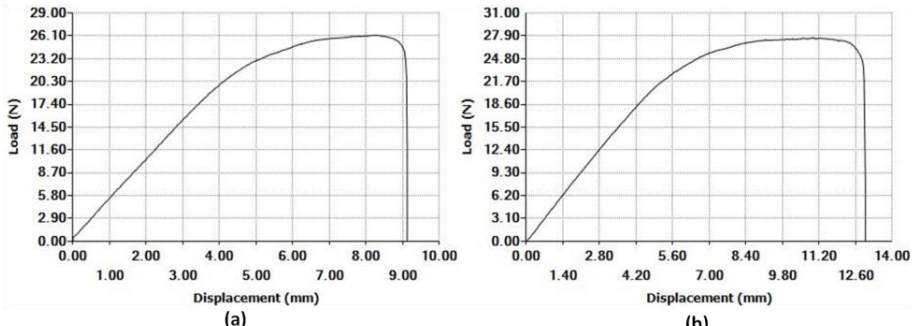

(b)

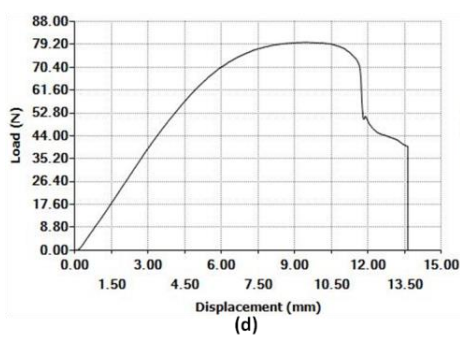

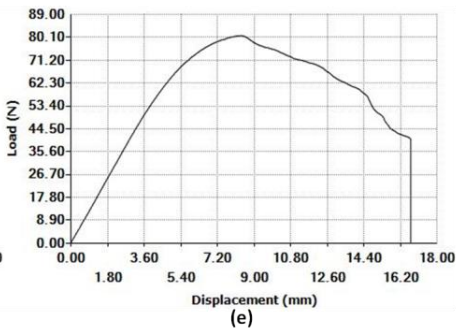

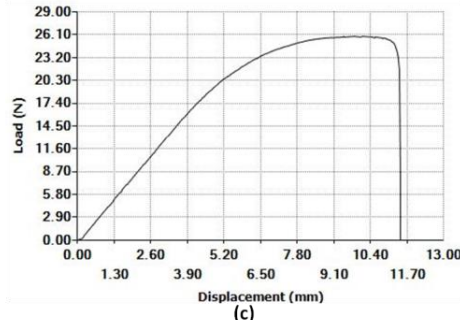

(c)
isplacement (mm)

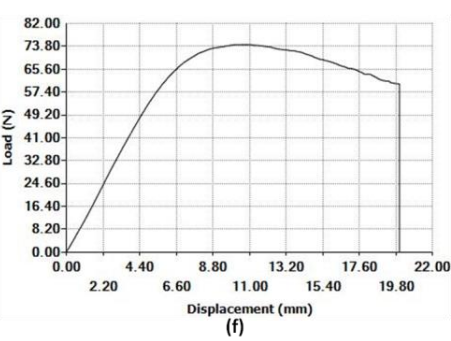

Fig. 11. Load Vs. displacement curves of flexural test for $80 \%$ infill density with (a-c) CF-ABS (d-f) PC-ABS

\section{Conclusions}

In this work, the mechanical properties of the CF-ABS and PC-ABS materials have been investigated and compared with respect to the varying FDM parameters like the infill geometry, infill percentage, and raster angle. The experiments have revealed that, compared to the CF-ABS material, PC-ABS material is stronger in compression, tension as well as in flexural at all the parametric settings. The infill percentage was observed to be proportional to the strength of the material as expected. The strength of the material was more sensitive to infill geometry in compression and flexural compared to that in tension. Triangular infill geometry was more strong in compression and flexural whereas grid infill geometry was strong in tension. 0-degree raster angle produced stronger material in all three criteria compared to the 45 and 90degree raster angle. The load vs displacement curves have been plotted to depict the maximum load and the behavior of the material in the elastic and plastic regions.

\section{References}

1. Boschetto, Alberto, and Luana Bottini. "Accuracy prediction in fused deposition modeling." The international journal of advanced manufacturing technology 73(5) 913 (2014)

2. De Laurentis, Kathryn J., and Constantinos Mavroidis. "Rapid fabrication of a non-assembly robotic hand with embedded components." Assembly Automation (2004).

3. Krache, Rachida, and Ismahane Debbah., Materials Sciences and Applications 2(5) 404 (2011)

4. Zhang, Hui, Zhong Zhang, and Claudia Breidt. Composites science and technology 64(13), 2021 (2004)

5. Liu, Yuan, and W. L. Yao. Int. J. Simul. Syst. Sci. Technol. 17, 25 (2016) 
6. Kamaal, M., M. Anas, H. Rastogi, N. Bhardwaj, and A. Rahaman, Progress in Additive Manufacturing 6(1), 63 (2021)

7. Kannan, Sridharan, and Manoharan Ramamoorthy. Materials Research Express 7(1), 015341 (2020)

8. Ning, Fuda, Weilong Cong, Yingbin Hu, and Hui Wang., Journal of Composite Materials 51(4), 451 (2017)

9. Cantrell, Jason T., Sean Rohde, David Damiani, Rishi Gurnani, Luke DiSandro, Josh Anton, Andie Young, Rapid Prototyping Journal 15(1), 2004 (2017)

10. Uddin, M. Sidek, M. F. R. Sidek, M. A. Faizal, Reza Ghomashchi, and Alokesh Pramanik., Journal of Manufacturing Science and Engineering 139(8), 081018 (2017)
11. Fernandez-Vicente, Miguel, Wilson Calle, Santiago Ferrandiz, and Andres Conejero., $3 D$ printing and additive manufacturing 3(3), 183 (2016)

12. Rankouhi, Behzad, Sina Javadpour, Fereidoon Delfanian, and Todd Letcher., Journal of Failure Analysis and Prevention 16(3), 467 (2016)

13. Christiyan, KG Jaya, U. Chandrasekhar, and K. Venkateswarlu., Materials Science and Engineering, 114(1), 012109 (2016)

14. Durgun, Ismail, and Rukiye Ertan. "Experimental investigation of FDM process for improvement of mechanical properties and production cost." Rapid Prototyping Journal (2014)

15. Hill, Nevin, and Mehrdad Haghi. "Deposition direction-dependent failure criteria for fused deposition modeling polycarbonate." Rapid Prototyping Journal (2014) 